\title{
Diagnóstico de Falhas em um Sistema de Tanques Acoplados Operando em Múltiplas Regiões
}

\author{
Laila Sindra Ribeiro* Davi Fiorese Bissoli ** \\ Celso José Munaro*** \\ * Engenharia Elétrica, Universidade Federal do Espírito Santo, ES \\ (e-mail: laila.sindra@gmail.com). \\ ** Engenharia Elétrica, Universidade Federal do Espírito Santo, ES \\ (e-mail: davi.f.bissoli@gmail.com). \\ *** Departamento de Engenharia Elétrica, Universidade Federal do \\ Espírito Santo, ES (e-mail: cjmunaro@gmail.com).
}

\begin{abstract}
In this work, a methodology for fault detection and diagnosis in systems whose variables are non-stationary but vary continuously in an operation region is evaluated. The external analysis method was evaluated estimating different models to generate residues used for detection and diagnosis. Fault detection was performed using principal component analysis followed by monitoring with Hotteling's $\mathrm{T}^{2}$ statistics, quadratic prediction error and combined. Different classifiers were used for diagnosis using different hyperparameters and features. The methodology was applied to a four coupled tanks system subjected to 9 faults manifested in 4 different ways. The good results demonstrated that the methodology has good generalization capacity for treating failures in large operation regions without having been trained for all of them.

Resumo: Neste trabalho é avaliada uma metodologia para detecção e diagnóstico de falhas em sistemas cujas variáveis são não estacionárias, mas variam continuamente em uma região de operação. O método de análise por variáveis externas foi avaliado estimando diferentes modelos para geração dos resíduos usados para detecção e diagnóstico. A detecção de falhas foi feita usando análise de componentes principais seguida do uso de monitoramento com as estatísticas $\mathrm{T}^{2}$ de Hotteling, erro de predição quadrático e combinada. Para o diagnóstico foram usados diferentes classificadores, avaliados com diferentes hiperparâmetros e features. A metodologia foi aplicada a um sistema de quatro tanques acoplados sujeito a 9 falhas que se manifestam de 4 formas diferentes. Os bons resultados demonstraram que a metodologia tem boa capacidade de generalização por tratar falhas em grandes regiões de operação sem ter sido treinada para todas elas.
\end{abstract}

Keywords: Fault detection; Principal component analysis; Fault diagnosis; Quadruple tank process.

Palavras-chaves: Detecção de falhas; Análise de componentes principais; Diagnóstico de falhas; Sistema de tanques acoplados.

\section{INTRODUÇÃO}

A aplicação de monitoramento estatístico multivariável em conjunto de algoritmos de aprendizado de máquinas tem resultado em algoritmos com grande potencial para a detecção e diagnóstico de falhas em processos industriais. Entretanto, tarefas tais como o gerenciamento de eventos anormais muitas vezes ainda são executadas por operadores a partir de seu conhecimento sobre o processo. A rápida detecção e diagnóstico de falhas evita danos aos equipamentos, paradas de produção e queda na qualidade do produto (Venkatasubramanian et al., 2003).

Métodos de monitoramento de falhas baseados em dados têm sido objeto de muitas pesquisas na literatura atualmente. Processos industriais possuem geralmente muitas variáveis, de modo que técnicas de redução de dimensionalidade como Análise por Componentes Principais (PCA) e Mínimos Quadrados Parciais (PLS) são alternativas usuais. As variáveis podem ter distribuição Gaussiana ou NãoGaussiana. As técnicas mais tradicionais envolvem o controle de processos com estatísticas multivariadas (MSPC) via $\mathrm{PCA}$ seguido de monitoramento com estatística $\mathrm{T}^{2}$ de Hotteling e/ou erro de previsão quadrático (SPE). Caso as variáveis não tenham distribuição Gaussiana, técnicas tais como Análise de Componentes Individuais (ICA), Modelos de Mistura Gaussiana (GMM) e Descrição de Dados por Vetores Suporte (SVDD) podem ser usadas. Ao leitor interessado, essas técnicas são descritas em Aldrich e Auret (2013).

Caso as relações entre as variáveis sejam não lineares, as técnicas tradicionais de MSPC baseadas em PCA não 
se aplicam. Neste caso, o uso de Redes Neurais Artificiais (ANN) para criar um modelo PCA não-linear, uso de métodos baseados em kernel e uso de métodos com aproximação linear por partes (o GMM por exemplo) são alternativas.

As técnicas tradicionais de MSPC baseadas em PCA consideram em geral apenas um ponto de operação. Quando esta condição não é satisfeita, o uso de Análise por Variáveis Externas (VE) proposta por Kano et al. (2004) é uma alternativa. Para processos com mudanças lentas é possível utilizar PCA adaptativo ou PLS recursivo. Em alguns casos, é fundamental monitorar falhas durante a transição entre pontos de operação, nestes casos métodos de modelagem suave, em geral com uso de múltiplos modelos probabilísticos são utilizadas. Mais informações sobre as diversas técnicas para detecção de falhas de acordo com as características dos dados de processo, bem como uma comparação das vantagens e desvantagens de cada uma podem ser encontrados em Ge et al. (2013).

Para o diagnóstico de falhas é possível utilizar classificadores como Redes Neurais Artificiais (ANN), K-vizinhos Próximos (KNN), Árvore de Decisão (DT), Máquina de Vetores Suporte (SVM), ou outros, treinados a partir de dados. Jung et al. (2018) comparou o desempenho dos classificadores SVM, KNN ponderado e DT, testados em um protótipo construído em laboratório para representar um sistema de combustível de veículos aéreos não tripulados (UAV). Também é possível utilizar inferência Bayesiana para diagnosticar falhas, como em Ge e Song (2009) e Md. Tanjin Amin (2018).

Neste trabalho foi avaliada a detecção e diagnóstico de falhas em um sistema de tanques acoplados, utilizando o software MATLAB e Simulink para simular um sistema semelhante ao proposto em Alvarado et al. (2006). Foi avaliado o uso de diversos modelos para análise de VE, PCA aplicado aos resíduos desses modelos, monitoramento com estatística $\mathrm{T}^{2}$, SPE e combinada, e diagnóstico com classificadores DT, SVM, KNN e ANN, analisando o efeito da alteração de hiperparâmetros e features utilizadas nos classificadores. A contribuição deste trabalho reside na comparação entre as técnicas mencionadas acima, avaliando seu desempenho com base no tempo de detecção da falha e acurácia, aplicando a um estudo de caso.

Nas seções seguintes, o leitor encontrará uma breve revisão teórica das técnicas utilizadas, seguida da descrição do sistema de quatro tanques, da metodologia utilizada para realizar os testes e os resultados obtidos.

\section{REVISÃO TEÓRICA}

\subsection{PCA e estatísticas $T^{2}$, SPE e Combinada}

Em processos com variáveis altamente correlacionadas, como um sistema de tanques acoplados, um pequeno conjunto de componentes principais pode ser usado mantendo boa parte da variância dos dados. A análise por componentes principais é usada para reduzir a dimensionalidade dos dados e aumentar a robustez do cálculo da estatística, pois as componentes menos relevantes de conjuntos de dados modelam majoritariamente ruídos dos sensores (MacGregor e Kourti, 1995).
Para construção de um modelo PCA, toma-se para treinamento um conjunto de variáveis $X \operatorname{com} n$ amostras e $k$ variáveis que caracterizam operação normal do processo. A partir desse conjunto retira-se sua média e calcula-se a matriz de covariância $S$, dada por

$$
S=\frac{1}{n-1} X^{T} X=V \Lambda V^{T}+\tilde{V} \tilde{\Lambda} \tilde{V}^{T}
$$

onde $V^{k \times a}$ é a matriz de carregamento com $a$ componentes principais associadas aos maiores autovalores de $S$ e $\Lambda$ é a variância dos dados mantidos por estas componentes.

Para cada nova amostra gerada $x$ das variáveis, aplicase uma das estatísticas definidas para monitoramento, sendo essas $T^{2}$ de Hotelling, Squared Prediction Error (SPE) e Combinada ( $T^{2}$ e SPE). O resultado do cálculo das estatísticas é então comparado com um limiar dado por distribuições estatísticas de acordo com um nível de confiança previamente definido.

A estatística $T^{2}$ tem seu limiar definido pela distribuição $F$ e o nível de confiança determinado. A estatística SPE está associada ao desvio das observações em relação à sua projeção no espaço reduzido do modelo PCA. Seu limiar bem como da estatística Combinada são detalhados em Alcala e Qin (2009).

\subsection{Análise por Variáveis Externas}

Para que os métodos de monitoramento clássicos baseados em PCA possam ser usados para detecção e diagnóstico de falhas é necessário que as variáveis permaneçam em estado estacionário durante a normalidade. Mudanças no ponto de operação são tratados como falhas. Uma alternativa da literatura para este caso é o uso de múltiplos modelos gaussianos (Ge e Song, 2009), que requerem a escolha dos modelos para o conjunto de dados e o uso de pesos para ponderar os modelos para cada nova amostra, sendo de difícil implementação.

Uma alternativa foi o método de análise de variáveis externas proposto em Kano et al. (2004). A utilização desse método possibilita distinguir falhas de mudanças nas condições de operação, desde que as mudanças ocorram apenas devido a variáveis conhecidas, denominadas variáveis externas.

O conjunto de dados $X$ com $m$ variáveis e $n$ amostras é decomposto em dois conjuntos de variáveis $H$ e $G$, que representam o grupo de variáveis externas e o grupo de variáveis principais monitoradas, respectivamente. A relação entre os dois conjuntos de variáveis é dada por uma matriz de coeficientes $C$ que pode ser obtida via mínimos quadrados. Com esta matriz e a equação 2 calcula-se o resíduo entre as variáveis monitoradas e sua estimativa obtida a partir das variáveis externas. Este resíduo será usado para o monitoramento de falhas.

$$
E=H-G C
$$

Neste trabalho os resíduos também serão calculados via regressão por vetores de suporte (SVR), redes neurais de regressão generalizada (GRNN) e regressões por árvores de decisão. 


\subsection{Métodos de classificação para diagnóstico}

Nesse trabalho utilizaremos técnicas supervisionadas de classificação, usando dados rotulados para aprender uma função de classificação. Em Aldrich e Auret (2013) podese encontrar definições para alguns classificadores além de outras informações sobre MSPC.

- KNN: É um processo de agrupamento que cria grupos com características similares (Denoeux, 1995). Inicializam-se centros de grupos aleatoriamente, objetos são alocados aos centros mais próximos, os centros são recalculados e repete-se esse processo até que não haja mudanças significativas no modelo.

- Árvore de Decisão (DT): São modelos estatísticos que utilizam um conjunto de treinamento para a classificação de dados através da estratégia dividire-conquistar. É feita a decomposição de um problema complexo em sub-problemas mais simples e reaplicada essa técnica recursivamente (Gama, 2004).

- Redes Neurais Artificiais (ANN): São compostas por elementos computacionais distribuídos em várias camadas. Os pesos entre seus nós se adaptam durante o processo de aprendizagem.

- Máquina de Vetores Suporte (SVM): Proposta por Cortes e Vapnik (1995), a SVM foi concebida para a resolução de problemas de classificação binária e funciona de forma à encontrar uma função (hiperplano) que separe as diferentes classes minimizando seu erro.

\section{ESTUDO DE CASO: SISTEMA DE TANQUES ACOPLADOS}

O sistema de quatro tanques acoplado foi originalmente proposto por Johansson et al. (1999) para estudos de controle multivariado com sistemas de fase não-mínima e usado aqui para detecção e diagnóstico de falhas. A Figura 1 ilustra o sistema utilizado, que é uma adaptação feita do modelo proposto em Alvarado et al. (2006). Este sistema também foi utilizado em Bissoli e Munaro (2018) para detecção e diagnóstico de falhas, onde os autores apresentaram uma descrição de alguns tipos de falhas que podem ser encontradas neste sistema e testaram algoritmos em 5 pontos de operação. Neste trabalho, estudou-se a detecção e diagnóstico de falhas com o ponto de operação variando continuamente. Esta situação é comum quando as referências das malhas de controle são dadas por otimizadores. Esta abordagem permite treinar modelos nos múltiplos pontos de operação e avaliar a eficácia da detecção e do diagnóstico com falhas ocorrendo em qualquer região de operação.

O modelo físico que representa o comportamento do sistema da Figura 1 é apresentado nas equações 3-6, obtido a partir da lei de Bernoulli e do balanço de massas. Nas equações, $c_{i}$ é valor de abertura da válvula $i$ do sistema no intervalo de 0 a $100 \%, a_{i}$ é a área do cano de saída do tanque $i \mathrm{em} \mathrm{cm}{ }^{2}, A_{i}$ é a área do tanque $i \mathrm{em} \mathrm{cm}^{2}, h_{i}$ é o nível do tanque $i, y_{i}$ é a abertura da válvula bidirecional que determina a razão de vazão para o tanque superior e inferior, $q_{i}$ é a vazão proveniente da bomba $i$. Por fim, $g$ é a constante de aceleração da gravidade.

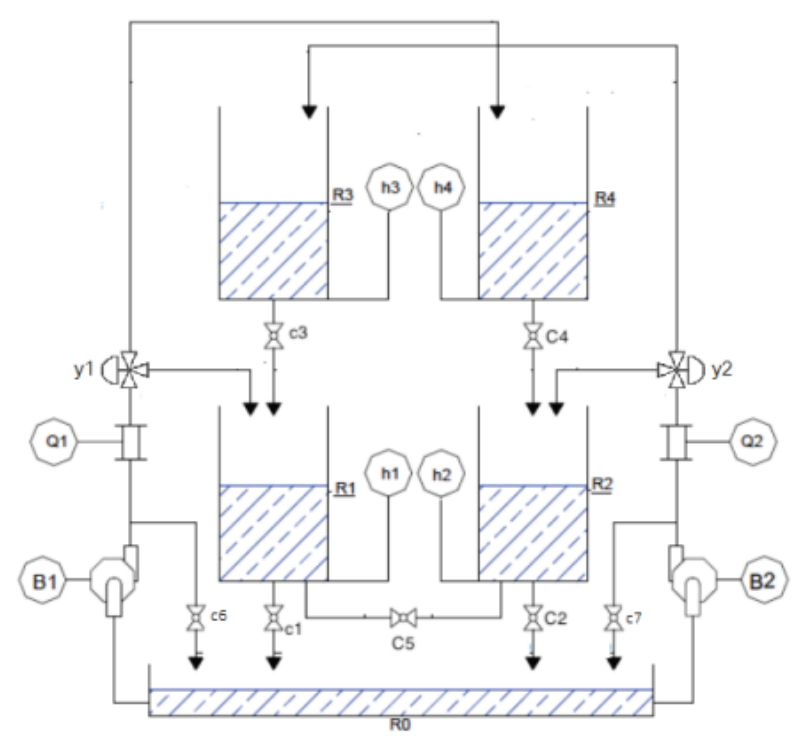

Figura 1. Sistema de tanques acoplados utilizado. Fonte: (Bissoli e Munaro, 2018).

\subsection{Descrição das Falhas}

Os níveis dos reservatórios $\mathrm{R} 1$ e $\mathrm{R} 2$ operam em malha fechada, controlados pelas vazões Q2 e Q1, respectivamente. Foram avaliadas 9 falhas no sistema de tanques, manifestadas de 4 formas diferentes: um aumento abrupto da falha usando um degrau positivo (DP) ou negativo (DN), um aumento(RP)/diminuição(RN) gradual da falha usando uma rampa. As 9 falhas estão descritas na Tabela 1. Estes tipos de falhas representam falhas que ocorrem gradualmente ou de forma rápida, comum em vazamentos e mudança no offset de medidores, por exemplo.

\subsection{Coleta de dados}

A simulação foi feita no software MATLAB e Simulink, com o setpoint (SP) de $\mathrm{H} 1$ e de $\mathrm{H} 2$ variando de forma senoidal com dinâmica mais lenta do que a dinâmica do sistema. Foram coletadas 5501 amostras em funcionamento normal para treinar um modelo para monitoramento e outras 5501 amostras para teste do monitoramento em condições normais. Para simulações com inclusão de falhas, foram coletados 2 conjuntos de dados, um para treino e um para teste. Cada conjunto possui 36 subconjuntos (um para cada falha e seus 4 tipos de falha) com 100 amostras em condições normais e 100 amostras em falha. A taxa de amostragem foi de 1 amostra por segundo, usualmente a menor taxa utilizada em processos industriais. A Figura 2 apresenta as 8 variáveis monitoradas em condições de operação normal que foram utilizadas para treinamento do modelo usado para detecção de falha.

\section{METODOLOGIA}

\subsection{Geração de resíduos com variáveis externas}

Para remover o efeito da mudança das referências (variáveis externas SP de H1 e SP de H2) sobre as variáveis principais (níveis $\mathrm{H} 1, \mathrm{H} 2, \mathrm{H} 3, \mathrm{H} 4$ e as vazões Q1 e Q2) foram testados modelos de SVR, DT, Conjunto de Árvores 


$$
\begin{gathered}
\frac{d h_{1}}{d t}=-c_{1} \cdot \frac{a_{1}}{A_{1}} \sqrt{2 g h_{1}}+\left(1-y_{1}\right) \frac{q_{1}}{A_{1}}+c_{3} \cdot \frac{a_{3}}{A_{1}} \sqrt{2 g h_{3}}+\operatorname{sign}\left(h_{2}-h_{1}\right) c_{5} \cdot \frac{a_{1}}{A_{1}} \sqrt{2 g\left|h_{2}-h_{1}\right|} \\
\frac{d h_{2}}{d t}=-c_{2} \cdot \frac{a_{2}}{A_{2}} \sqrt{2 g h_{2}}+\left(1-y_{2}\right) \frac{q_{2}}{A_{2}}+c_{4} \cdot \frac{a_{4}}{A_{2}} \sqrt{2 g h_{4}}+\operatorname{sign}\left(h_{1}-h_{2}\right) c_{5} \cdot \frac{a_{1}}{A_{2}} \sqrt{2 g\left|h_{1}-h_{2}\right|} \\
\frac{d h_{3}}{d t}=-c_{3} \cdot \frac{a_{3}}{A_{3}} \sqrt{2 g h_{3}}+y_{2} \frac{q_{2}}{A_{3}} \\
\frac{d h_{4}}{d t}=-c_{4} \cdot \frac{a_{4}}{A_{4}} \sqrt{2 g h_{4}}+y_{1} \frac{q_{1}}{A_{4}}
\end{gathered}
$$

Tabela 1. Falhas aplicadas ao sistema de tanques

\begin{tabular}{|c|c|}
\hline Falha & Descrição \\
\hline 1 & Alteração da eficiência da Bomba 1 \\
\hline 2 & Alteração da eficiência da Bomba 2 \\
\hline 3 & $\begin{array}{c}\text { Alteração na vazão de R1 } \\
\text { (Vazamento ou Entupimento) }\end{array}$ \\
\hline 4 & $\begin{array}{c}\text { Alteração na vazão de R2 } \\
\text { (Vazamento ou Entupimento) }\end{array}$ \\
\hline 5 & $\begin{array}{c}\text { Alteração na vazão de R3 } \\
\text { (Vazamento ou Entupimento) }\end{array}$ \\
\hline 6 & $\begin{array}{c}\text { Alteração na vazão de R4 } \\
\text { (Vazamento ou Entupimento) }\end{array}$ \\
\hline 7 & $\begin{array}{c}\text { Alteração do acoplamento } \\
\text { entre os tanques }\end{array}$ \\
\hline 8 & Offset na medição no sensor de nível H1 \\
\hline 9 & Offset na medição no sensor de nível H2 \\
\hline
\end{tabular}
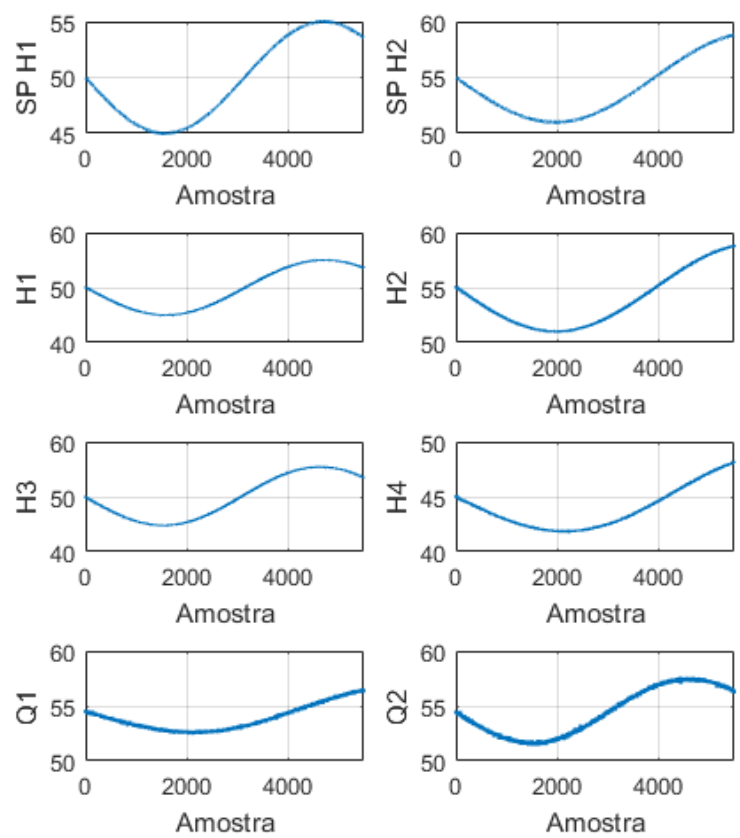

Figura 2. Comportamento das variáveis em condições normais.

de Decisão, Modelos Polinomiais de $1^{a}$ até $3^{a}$ ordem e Rede Neural de Regressão Generalizada (GRNN). Comparou-se a norma dos resíduos obtidos dos diversos modelos usando os dados de teste. Os modelos GRNN e polinomial de $1^{a}$ ordem (modelo linear) foram utilizados para o monitoramento, com o objetivo de comparar o desempenho entre estes modelos. Para o GRNN, o critério de menor norma dos resíduos nos dados de teste foi utilizado para escolher o hiperparâmetro de suavização.

\subsection{Detecção de falhas}

De posse do modelo para gerar os resíduos, construiuse um modelo para monitoramento estatístico de PCA, usando como critério de escolha do número de componentes principais manter no mínimo $95 \%$ da variância dos dados. Calculou-se então os limiares para $95 \%$ de confiança para as estatísticas $\mathrm{T}^{2}$, SPE e Combinada. Foi considerado o valor de 5 ultrapassagens consecutivas do limiar como feito em Ge et al. (2008) para mensurar o tempo para detecção da falha. Comparou-se a eficiência de detecção das estatísticas $\mathrm{T}^{2}$, SPE e combinada medindo-se o tempo de detecção para cada uma das 9 falhas e cada tipo (degrau e rampa positivos e negativos).

\subsection{Diagnóstico de falhas}

Para o diagnóstico de falhas foram comparados os resultados dos classificadores DT, SVM, KNN e ANN, variando hiperparâmetros. Após a detecção da falha, são calculadas características (features) dos resíduos usando uma janela deslizante: desvio em relação à média $(\mathrm{dv})$, desvio absoluto em relação a média (dva), desvio padrão (dp) e análise de componentes principais (pca). Também foi testado a utilização de mais de um tratamento, aumentando o número de características nos classificadores e comparando o desempenho através da acurácia obtida com os dados de teste. Para os classificadores KNN e ANN avaliou-se o efeito da variação do número de vizinhos e da suavização, respectivamente, e para ambos avaliou-se o efeito do número de amostras de cada falha e tipo utilizadas para treino do classificador.

\section{APLICAÇÃO E RESULTADOS}

\subsection{Geração de resíduos com VE}

A norma dos resíduos obtidos nos conjuntos de treino e teste está apresentada na Tabela 2. Como os modelos polinomial e GRNN apresentaram os melhores resultados, os resíduos destes modelos foram utilizados para aplicação de PCA e monitoramento. Os resíduos obtidos nos dados de teste para os modelos GRNN e linear são apresentados na Figura 3.

\subsection{Detecção de falhas}

Após verificar que os resíduos gerados seguem distribuição normal, aplicou-se PCA mantendo 95\% da variância dos dados, o que resultou em 4 componentes principais. Monitorou-se os dados com as estatísticas $\mathrm{T}^{2}$, SPE e combinada. No trecho da amostra 1000 até 2000 da Figura 3a observa-se que os resíduos refletem o formato do SP, 
Tabela 2. Norma dos resíduos utilizando diferentes modelos para VE

\begin{tabular}{|c|c|c|c|c|c|c|c|}
\hline Norma dos & \multirow{2}{*}{$\begin{array}{c}\text { SVR } \\
\text { resíduos }\end{array}$} & DT & \multirow{2}{*}{$\begin{array}{c}\text { Conjunto } \\
\text { de árvores }\end{array}$} & \multicolumn{2}{|c|}{ Polinomial de ordem } & \multirow{2}{*}{$\begin{array}{c}\text { GRNN } \\
\text { Suavização 0,35 }\end{array}$} \\
\hline Treino & 45,95 & 39,55 & 6,07 & 8,20 & 7,80 & 7,79 & 7,94 \\
\hline Teste & 45,88 & 39,61 & 8,98 & 8,35 & 7,95 & 7,95 & 8,01 \\
\hline
\end{tabular}

Tabela 3. Tempos de detecção de falhas com estatística $\mathrm{T}^{2}$.

\begin{tabular}{|c|c|c|c|c|c|c|c|c|c|c|}
\hline & & \multicolumn{10}{|c|}{ Falha } \\
\cline { 2 - 12 } & & 1 & 2 & 3 & 4 & 5 & 6 & 7 & 8 & 9 \\
\hline \multirow{3}{*}{ Tipo de } & DN & 108 & 108 & 108 & 108 & 108 & 108 & 108 & 107 & 107 \\
\cline { 2 - 12 } & DP & 108 & 108 & 108 & 108 & 107 & 108 & 108 & 107 & 107 \\
\cline { 2 - 11 } & RN & 119 & 118 & 112 & 113 & 110 & 111 & 116 & 108 & 108 \\
\cline { 2 - 11 } & RP & 116 & 118 & 111 & 111 & 112 & 112 & 115 & 109 & 109 \\
\hline
\end{tabular}

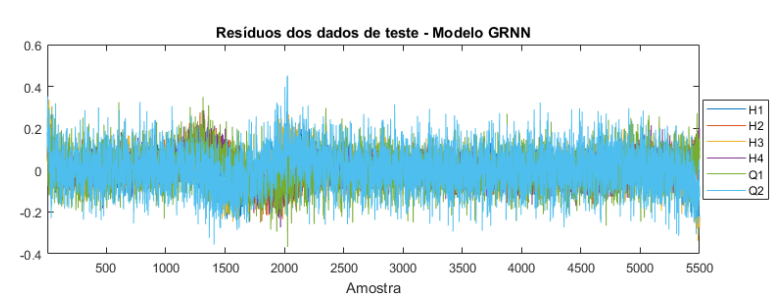

(a) Resíduos modelo GRNN.

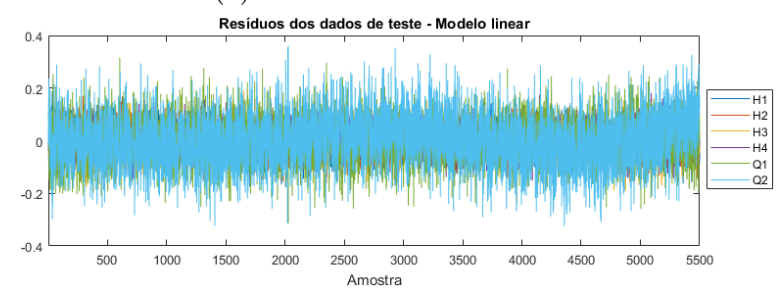

(b) Resíduos modelo linear.

Figura 3. Resíduos em dados de teste após VE.

indicando que estes ainda sofrem muita influência do ponto de operação. Isto é perceptível nas estatísticas sob a forma de violações do limiar neste trecho. Portanto, os melhores resultados foram obtidos utilizando o modelo linear. A estatística $\mathrm{T}^{2}$ resultou em menores tempos de detecção de falha e com número esperado de falsos positivos no trecho em operação normal (aproximadamente 5\%). A Figura 4 mostra as variáveis monitoradas, os resíduos do modelo linear e o monitoramento com estatística $\mathrm{T}^{2}$ para falha $1 \mathrm{em}$ rampa positiva $(\mathrm{RP})$. Desta Figura observa-se que a falha ocorrida após a amostra 100 é detectada poucos segundos depois de seu início. Os tempos de detecção para cada falha e cada tipo são apresentados na Tabela 3. Observase que o maior tempo de detecção ocorre para a falha $1 \mathrm{em}$ rampa positiva e ocorre no instante 119 monitorando com $\mathrm{T}^{2}$. Utilizando as estatísticas SPE e combinada, o maior tempo de detecção ocorre para as falhas 1 e 2 em rampa negativa na amostra 121 . Os outros tempos obtidos com estas estatísticas serão omitidos devido ao pouco espaço disponível neste artigo.

\subsection{Diagnóstico de falhas}

A acurácia obtida com os diversos classificadores aplicados aos dados de treino e de teste, de acordo com o tipo de feature utilizada na classificação é mostrada na Tabela 4. São apresentados os resultados para as features: dv, dva, dp calculadas em uma janela deslizante de 20 amostras, pca e a utilização de mais de uma feature, onde a coluna "Todos" corresponde ao uso de dv, dva, dp e pca. Foram utilizadas 30 amostras de cada falha e cada tipo para treinamento dos classificadores. Dentre os valores de hiperparâmetros testados para os classificadores KNN e para ANN o resultado apresentado na Tabela 4 foi o que alcançou melhor acurácia, sendo 1 vizinho como hiperparâmetro do KNN e para a ANN suavização de 0,001. Os classificadores com melhor desempenho foram KNN e ANN, que obtiveram acurácia de 94,58\% em dados de teste, destacado em verde na Tabela 4, utilizando todas as features e 30 amostras de cada falha e cada tipo para treinamento. Destaca-se que a variação da acurácia de acordo com a característica não se comporta da mesma maneira nos classificadores e que com exceção da DT, o aumento do número de features melhora a acurácia do classificador.

A fim de analisar a influência do número de amostras utilizadas no treino dos classificadores KNN e ANN sobre a acurácia da classificação, avaliou-se a acurácia em função do número de amostras de cada falha e tipo utilizado no treinamento destes classificadores, mantendo os outros hiperparâmetros utilizados anteriormente. A Figura 5 apresenta as acurácias obtidas, sendo que os classificadores comportam-se de maneira idêntica exceto para 55 e 60 amostras, onde o KNN atinge $100 \%$ de acurácia e o ANN atinge $99,95 \%$.
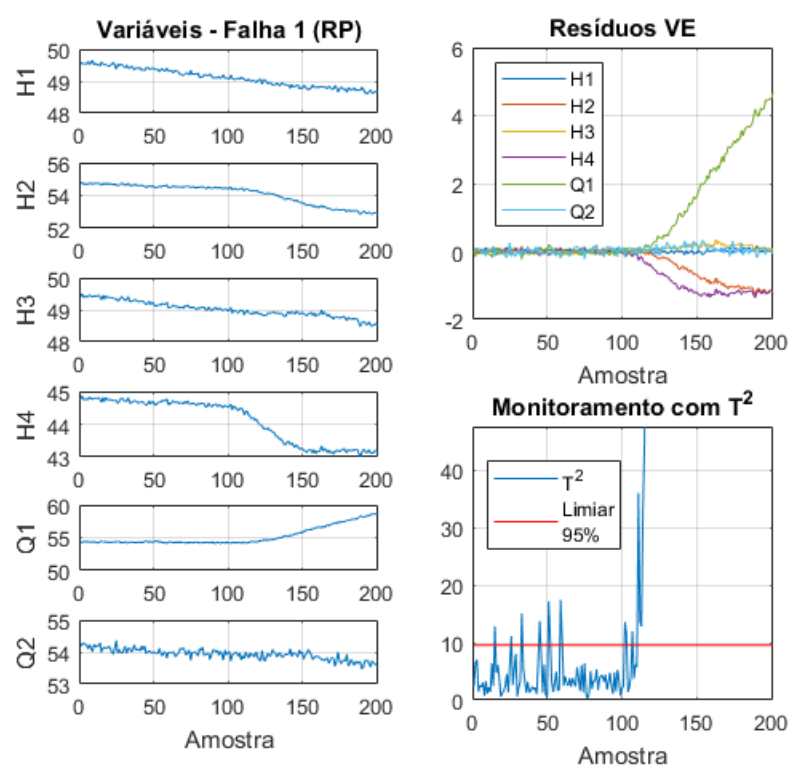

Figura 4. Variáveis, resíduos e monitoramento com estatística $T^{2}$ para falha 1 em rampa positiva. 
Tabela 4. Acurácia (em \%) obtida com os classificadores testados.

\begin{tabular}{|c|c|c|c|c|c|c|c|}
\hline \multicolumn{2}{|c|}{} & \multicolumn{7}{|c|}{ Features utilizadas para classificação } \\
\hline Classificador & Dados & $\mathrm{dv}$ & $\mathrm{dva}$ & $\mathrm{dp}$ & $\mathrm{pca}$ & $\mathrm{dp} \mathrm{e} \mathrm{dv}$ & Todos \\
\hline \multirow{2}{*}{$\mathrm{DT}$} & Treino & 96,30 & 97,69 & 11,11 & 80,74 & 96,30 & 97,87 \\
\cline { 2 - 8 } & Teste & 64,30 & 61,48 & 10,80 & 26,96 & 64,30 & 63,48 \\
\hline \multirow{2}{*}{ SVM } & Treino & 50,00 & 87,69 & 93,15 & 20,65 & 96,20 & 100,00 \\
\cline { 2 - 9 } & Teste & 46,36 & 67,28 & 70,58 & 18,04 & 76,59 & 85,93 \\
\hline \multirow{2}{*}{ KNN } & Treino & 100,00 & 100,00 & 100,00 & 100,00 & 100,00 & 100,00 \\
\cline { 2 - 8 } & Teste & 86,57 & 82,65 & 78,19 & 45,50 & 92,21 & 94,58 \\
\hline \multirow{2}{*}{ ANN } & Treino & 100,00 & 100,00 & 99,07 & 99,81 & 100,00 & 100,00 \\
\cline { 2 - 8 } & Teste & 79,80 & 74,38 & 76,73 & 41,12 & 92,12 & 94,58 \\
\hline
\end{tabular}

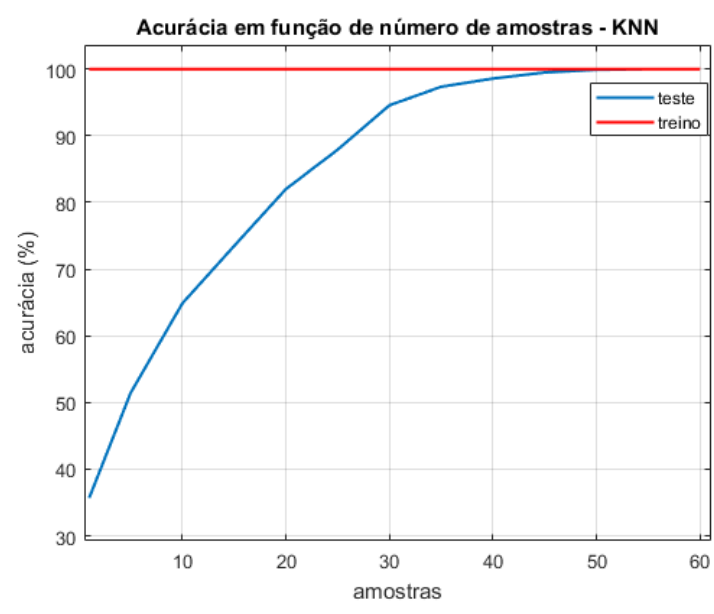

(a) KNN.

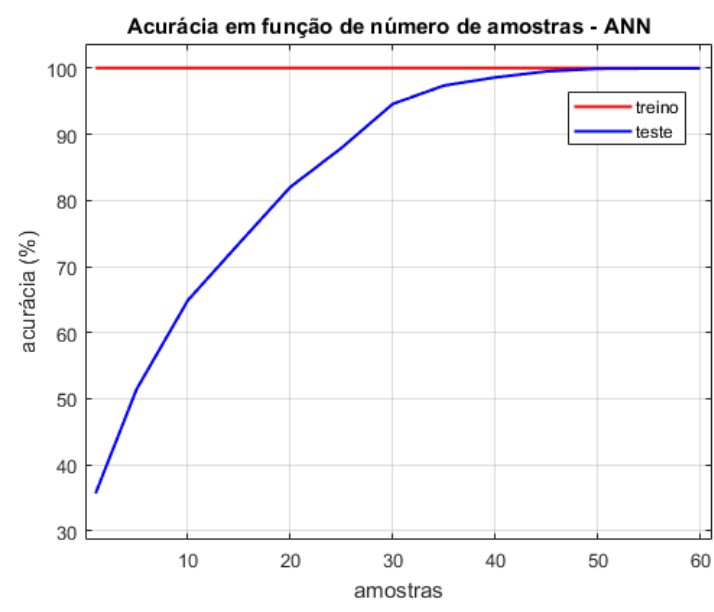

(b) ANN.

Figura 5. Acurácia em função do número de amostras para os classificadores KNN e ANN.

\section{CONCLUSÕES}

Neste trabalho, verificou-se que a análise de variáveis externas pode ser utilizada para gerar modelos cujos resíduos sejam adequados para aplicação de detecção de falhas usando análise de componentes principais, mesmo em um sistema cujo ponto de operação varia continuamente. O modelo de regressão que permitiu monitoramento mais adequado foi o polinomial de $1^{a}$ ordem. Para monitoramento, foram avaliadas as estatísticas $\mathrm{T}^{2}$, SPE e combinada, sendo que a estatística $\mathrm{T}^{2}$ foi a que possibilitou detecção de falhas no menor tempo. Para o diagnóstico das 9 falhas, foram testados os classificadores DT, SVM, KNN e ANN, variando seus hiperparâmetros bem como as features utilizadas e o número de amostras para treino do classificador. Para KNN e ANN variou-se o número de vizinhos e suavização, respectivamente. Observou-se que tanto os classificadores KNN e ANN obtiveram desempenho similar.

A metodologia proposta mostrou-se eficaz para o estudo de caso analisado. Um ponto forte da metodologia é o fato do treinamento para uma região servir para as demais, desde que o modelo que gera os resíduos continue válido. Seu ponto fraco é a necessidade de dados rotulados para todas as falhas e tipos de falhas, o que pode ser uma dificuldade em sistemas reais. Além disto, a metodologia considera que as falhas ocorrem isoladamente. Nos casos de falhas simultâneas, podem-se utilizar, por exemplo, técnicas híbridas utilizando rede bayesiana para identificar a causa raiz das falhas, como feito por Md. Tanjin Amin (2018).
Em trabalhos futuros, pretende-se testar a metodologia proposta em uma planta piloto já disponível.

\section{AGRADECIMENTOS}

Este trabalho recebeu o apoio da Fundação de Amparo à Pesquisa e Inovação do Espírito Santo (FAPES) e da Vale, sob o termo de cooperação 29501.412.19434.18122015.

\section{REFERÊNCIAS}

Alcala, C. F. e Qin, S. J. (2009). Unified analysis of diagnosis methods for process monitoring, IFAC Proceedings Volumes 42(8): 1007 - 1012. 7th IFAC Symposium on Fault Detection, Supervision and Safety of Technical Processes.

Aldrich, C. e Auret, L. (2013). Unsupervised Process Monitoring and Fault Diagnosis with Machine Learning Methods, 1 edn, Springer-Verlag London.

Alvarado, I., Limon, D., García-Gabín, W., Alamo, T. e Camacho, E. F. (2006). An educational plant based on the quadruple-tank process, IFAC Proceedings Volumes 39(6): $82-87$.

Bissoli, D. F. e Munaro, C. J. (2018). Detecção e diagnóstico de falhas em sistema de tanques acoplados, Congresso Brasileiro de Automática, João Pessoa-PB .

Cortes, C. e Vapnik, V. (1995). Support-vector networks, Machine Learning 20(3): 273-297.

Denoeux, T. (1995). A k-nearest neighbor classification rule based on dempster-shafer theory, IEEE transactions on systems, man, and cybernetics 25(5): 804-813.

Gama, J. (2004). Functional trees, Machine Learning 55(3): 219-250. 
Ge, Z. e Song, Z. (2009). Multimode process monitoring based on bayesian method, Journal of Chemometrics 23: $636-650$.

Ge, Z., Song, Z. e Gao, F. (2013). Review of recent research on data-based process monitoring, Industrial and Engineering Chemistry Research 52(10): 3543-3562.

Ge, Z., Yang, C., Song, Z. e Wang, H. (2008). Robust online monitoring for multimode processes based on nonlinear external analysis, Industrial and Engineering Chemistry Research 47(14): 4775-4783.

Johansson, K. H., Horch, A., Wijk, O. e Hansson, A. (1999). Teaching multivariable control using the quadruple-tank process, Conference on Decision and Control pp. 807-812.

Jung, M., Niculita, O. e Skaf, Z. (2018). Comparison of different classification algorithms for fault detection and fault isolation in complex systems, Procedia Manufacturing 19: 111-118.

Kano, M., Hasebe, S. e Hashimoto, I. (2004). Evolution of multivariate statistical process control: Application of independent component analysis and external analysis, Computers and Chemical Engineering 28: 1157-1166.

MacGregor, J. e Kourti, T. (1995). Statistical process control of multivariate processes, Control Engineering Practice 3(3): 403 - 414.

Md. Tanjin Amin, Syed Imtiaz, F. K. (2018). Process system fault detection and diagnosis using a hybrid technique, Chemical Engineering Science 189: 191-211. Venkatasubramanian, V., Rengaswamy, R., Yin, K. e Kavuri, S. N. (2003). A review of process fault detection and diagnosis part i: Quantitative modelbased methods, Computers and Chemical Engineering 27(3): 293-311. 